\title{
Prompt Magnetic Resonance Imaging, Key to Correct Management of Neurocysticercosis in Developing Countries: A Case Report from Zambia
}

\author{
Victor Mwanakasale $^{1^{*}}$, Nataliya Yakovlyeva ${ }^{2}$, Kapambwe M Kamanga ${ }^{1}$, Pipina V Matafwali ${ }^{1}$ \\ ${ }^{1}$ Copperbelt University, Michael Chilufya Sata School of Medicine, Ndola, Zambia \\ ${ }^{2}$ Ndola Teaching Hospital, Ndola, Zambia
}

*Corresponding Author: Dr Victor Mwanakasale (MD), Lecturer-Medical Parasitology, Copperbelt University, Michael Chilufya Sata School of Medicine, Ndola, Zambia.

\begin{abstract}
Neurocysticercosis is caused by the infection with the larval form of Taneiasolium, the pork tape. It is the leading cause of acquired epilepsy in developing countries. Diagnosis of this condition is based on findings in radiological imaging, especially Magnetic Resonance Imaging. This case report demonstrates the importance of availability of Magnetic Resonance Imaging in the correct management of Neurocysticercosis.
\end{abstract}

Abbreviations: CNS-Central Nervous System, CSF- Cerebral Spinal Fluid, CT-Computer Tomography, MRI-Magnetic Resonance Imaging, NCC-Neurocysticercosis, NTDs- Neglected Tropical Diseases, WHOWorld Health Organisation

Keywords: Neurocysticercosis, Prompt, Magnetic, Resonance, Imaging, Management, Seizures

\section{INTRODUCTION}

Neurocysticercosis (NCC) is a disease characterized by the parasitic invasion of the Central Nervous System (CNS) by the larval stage of the pork tapeworm, Taeniasolium. It is the most common parasitic disease of the CNS. The disease is a zoonosis and belongs to a group of diseases classified as Neglected Tropical Diseases (NTDs) by the World Health Organisation (WHO). Man acquires the infection by ingesting the eggs of the tapeworm in human faecal contaminated water or food [1]. Another route of acquiring infection is by direct infection from anus to mouth via contaminated fingers of a person infected with the adult tapeworms in form of autoinfection or person to person transmission [2]. The larva that hatches from the ingested eggs lodge predominantly in the brain, muscles, and other soft tissues [3]. In the brain the larva frequently lodge in the parenchyma but not infrequently can lodge inside the ventricles and subarachnoid space or a combination of these three [3]. Clinical presentation is dependent on the location of the larvae. There is a variable time interval between the point of infection and the onset of symptoms, ranging from 1 to 30 years. Most symptomatic patients are aged $15-40$ years. The condition has no gender or race predilection [4]. The clinical features include seizures that are non-specific. Seizures are the most common symptom of NCC in young adults in endemic areas. Other presentations include headache, hydrocephalus, altered mental status, neurological deficits, and Bruns syndrome which comprises fever, vomiting, and stiff neck [4]. The disease is endemic in central and South America, Asia, and Africa. Disease occurrence is related to poor sanitation and hygiene.

We present a case of a patient that was initially wrongly diagnosed for a non-specific bacterial, fungal and viral infection at a private hospital that lacked appropriate imaging facilities but later correctly diagnosed as NCC at a government hospital that had imaging facilities in Zambia, Southern Africa.

\section{THE CASE}

A 34 years old male patient presented with history of seizures, headache, general body, malaise at a private hospital in the city of Ndola, Copperbelt province, Zambia on $23^{\text {rd }}$ January 
2020. The patient was immediately admitted. Before any laboratory investigations could be done the patient's condition changed. He went into a confusion state. He also developed weakness of both lower limbs. The patient was commenced on Ceftriaxone, Imipenam, Fluconazole, and Acyclovir, the same day, for five weeks. The patient' condition continued to deteriorate with Glasgow coma scale dropping to $9 / 15$. On $28^{\text {th }}$ February 2020 the patient was transferred and admitted to Ndola Teaching Hospital, which is a government-run hospital in the same city. Immediately upon admission, a Magnetic Resonance Imaging (MRI) was requested and done on the patient in the hospital's Radiology Department. The Axil MRI images revealed multiple round-like small-sized lesions that showed irregular altered signal intensity lesions hyperintense on T2WI and
FLAIR and hypointense on T1WI in parietal lobes. There was perilesional oedema. Other lesions were seen in periventricular area thatwere also well defined and rounded. The signal intensity was similar to the Cerebral Spinal Fluid (CSF). The scolexwas not seen within the lesions. The Circle of Willis showed perivascular infiltration with hyperintense signal. The fourth and both lateral ventricles were dilated due to mild communicating Hydrocephalus. All the pathology described above is shown in Figures 1, 2, and 3, below. A diagnosis of Colloidal- Vesicular stage NCC was made. The patient was commenced on Albendazole at $15 \mathrm{mg} / \mathrm{kg}$ O.D for 2 weeks on $5^{\text {th }}$ March 2020. The patient made a remarkable improvement and was discharged from the hospital at the end of the treatment course.

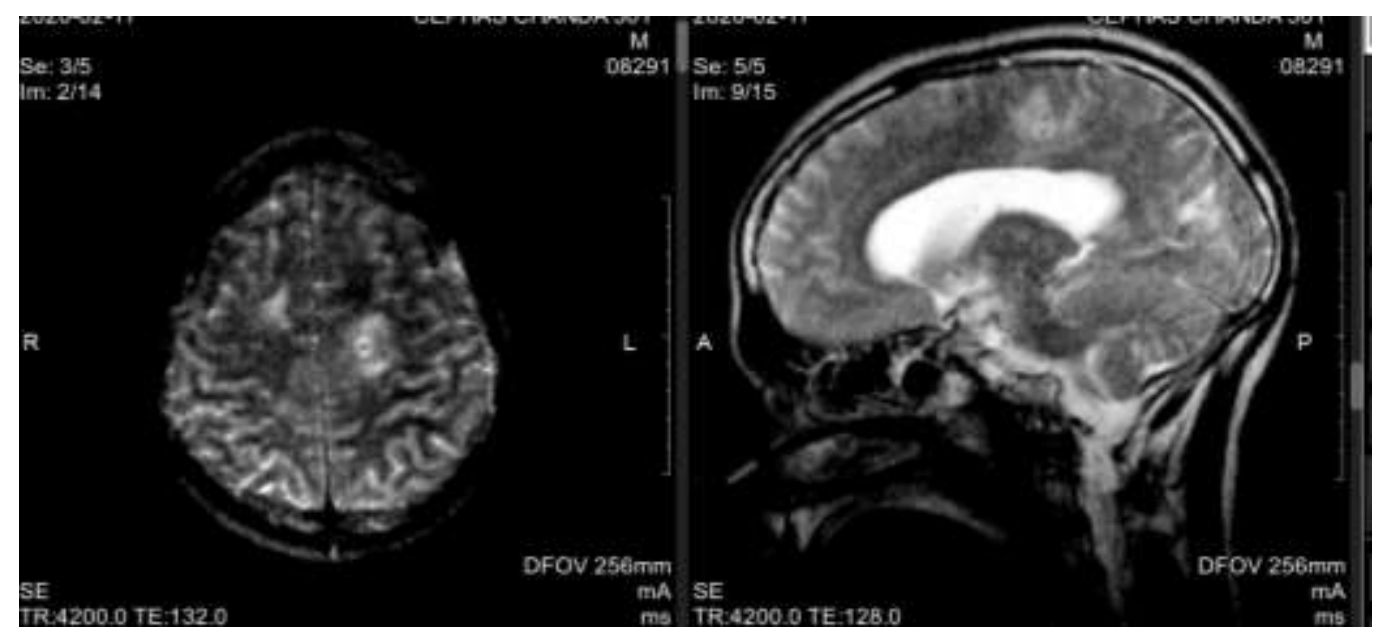

Figure 1. T2W axial and midsagittal planes. Multiple well-defined small-sized cysts seen in parietal and occipital lobe near the grey-white junction, adjacent to $4^{\text {th }}$ ventricle. The signal is hyperintense to CSF. Surrounding edema present. (At the time of examination, the contrast was not available)

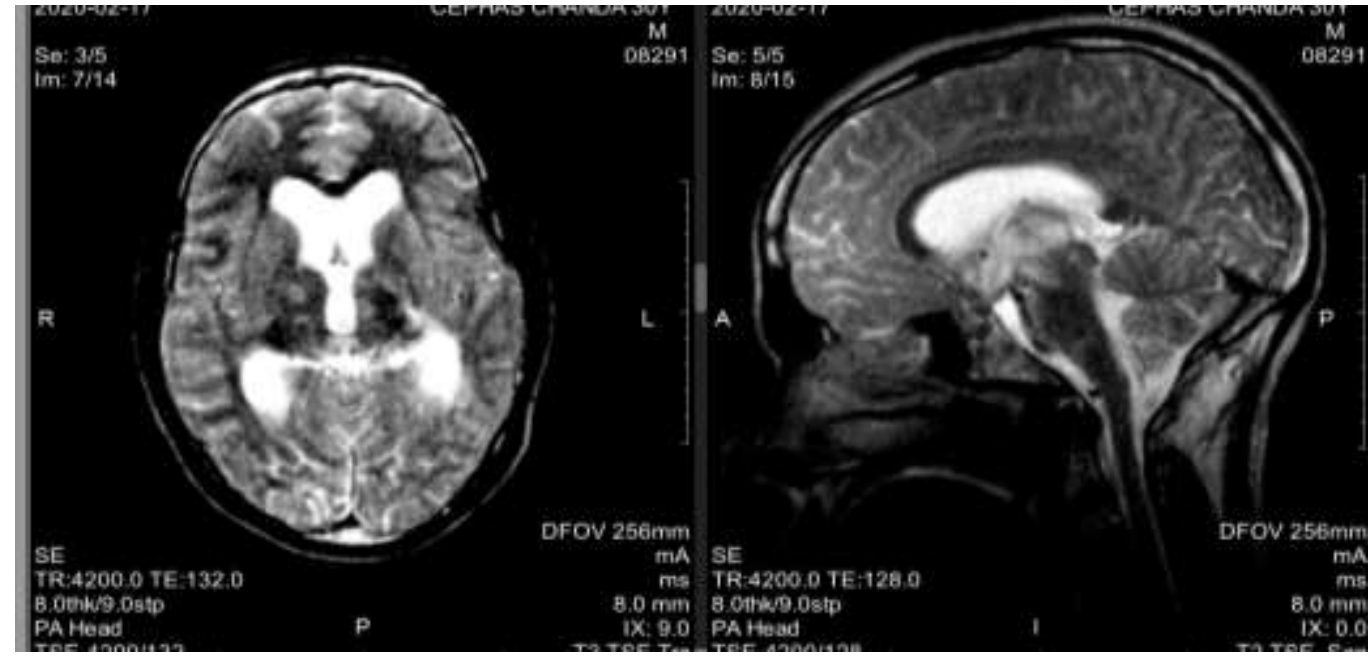

Figure 2. Same examination. T2W axial and sagittal plane images show small-sized grape-like (racemose) lesions in periventricular area and internal capsule. No visible scolex. Note the hyperintense signal in aqueduct zone, features of ventriculitis and moderate hydrocephalus. Prominent widened sulci evident 


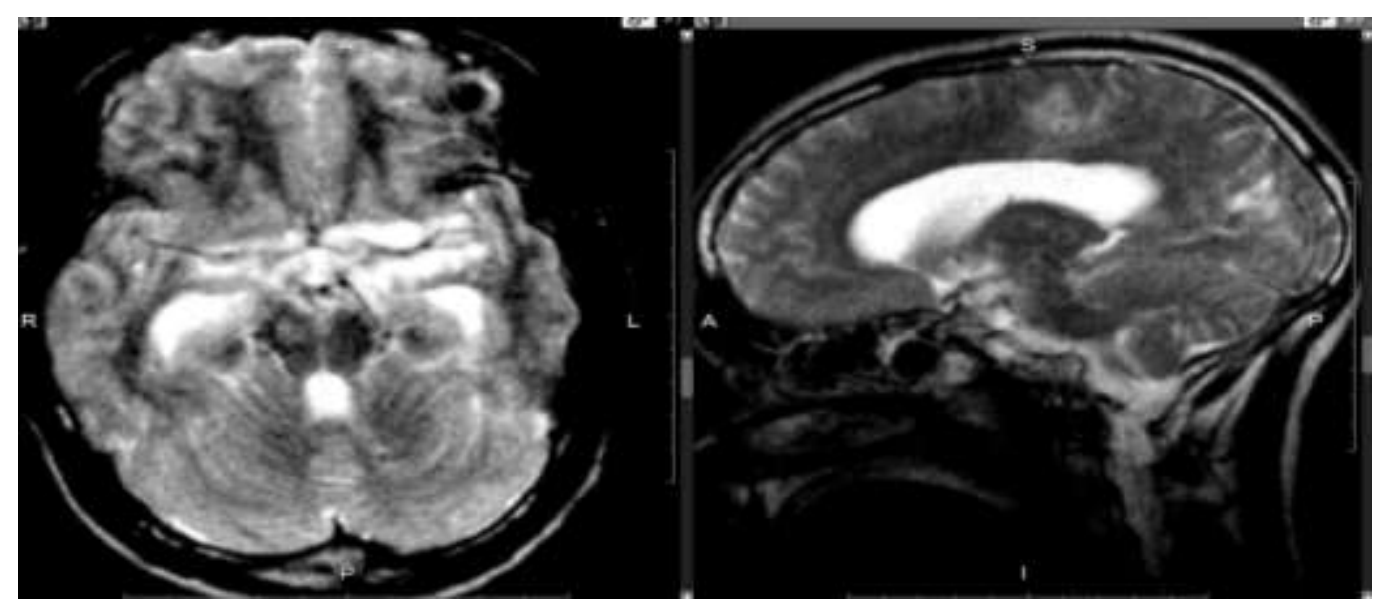

Figure 3. Same examination

\section{DISCUSSION}

In the diagnosis of NCC, the macroscopic appearance of the cysticerci varies according to their location within the CNS and the stage of the disease. Different stages of the disease and locations of the cysticerci may coexist [5]. NCC may be classified therefore according to location and stage of the disease. Cysticerci are classified, according to location, as Subarachnoid, Parenchymal, Ventricular, and Spinal. Based on the stage of the disease, cysticerci are classified as Non-cystic, Vesicular, Colloid-Vesicular, Granular-nodular, and calcified nodular. Our patient was diagnosed to be Colloidal-Vesicular stage of NCC. According to location, Subarachnoid location is the most common, followed by Parenchymal cysticerci. The third position in location is Ventricular cysticerci with the fourth ventricle being the most commonsite, followed by the lateral Ventricles.

The diagnosis of NCC is difficult because in many cases the infection with Taeniasolium cannot be demonstrated [6]. The diagnosis is based on a combination of epidemiological data, clinical presentation, radiological findings, and immunological tests such as serological tests for detection of anticysticercal antibodies in blood and Cerebral Spinal fluid. In developing countries where this disease is endemic, diagnosis is usually made on the clinical presentation in form of history of seizures and imaging results such as Computer Tomography (CT) and MRI findings. Under neuroimaging studies, CT findings are based on the identification of a well-defined cystic lesion in the cortical-juxtacortical junction, basal ganglia, cerebellum, midbrain, cisterns, or ventricular system. The lesion may show minimal contrast enhancement and an enhancing mural nodule, corresponding to the scolex. Chronic lesions of NCC appear as calcifications readily visible on CT images. In general, MRI is more sensitive than CT for the diagnosis of NCC. MRI allows the identification of a larger number of lesions, classify the stage of the disease, and determine the degree of inflammatory response by way of perilesional edema and blood-brain barrier disruption [6]. From a diagnostic standpoint and from the many imaging findings of NCC, only the presence of cystic lesions demonstrating the scolex is considered pathognomonic [7]. The scolex is visualized as a bright nodule within the cyst, producing the so-called "hole-with-dot" imaging that is seen in some vesicular cysts located in the brain parenchyma, the Subarachnoid space, or the Ventricular system [8]. Revised diagnostic criteria for NCC consider that cystic lesions showing the scolex on CT or MRI imaging are an absolute criterion for diagnosis of the disease, allowing its definite diagnosis [9].

Our case report has demonstrated what is already known, that the clinical features of NCC are non-specific. Hence, the key to the diagnosis of NCC is neuroimaging. Imaging techniques are not so readily available in endemic developing countries where they are most needed. In most endemic countries CT and MRI facilities are only available in few governmentrun hospitals. These imaging techniques are too expensive to be acquired by most private hospitals like in our case report. Therefore, cases of NCC are frequently misdiagnosed and mismanaged in most private and governmentrun hospitals lacking imaging facilities in endemic developing countries.

Differential diagnosis of NCC in endemic areas may be extremely difficult due to the coexistence of tuberculosis and other parasitic 
infections [10]. Ring-like lesions, solitary or multiple, in the parenchyma of the CNS are not specific for NCC and represent a diagnostic problem. In Colloidal-Vesicular NCC, like in our patient in the case report, the differential diagnosis should include tuberculomas, pyogenic abscesses, toxoplasmosis, Neurosyphilis, Hydatidosis, and primary and secondary neoplasms [11]. For calcified nodular stage, the differential diagnoses include carcinomas and amyloid microangiopathy [11]. Atypical presentation of NCC may mimic other neurological diseases [11]. Basal arachnoiditis may appear like carcinomatous meningitis, granulomatous diseases like Sarcoidosis, tuberculosis, and fungal meningitis [11]. The patient in our case report was initially treated for unconfirmed bacterial, viral, and fungal infections. For spinal NCC, the differential diagnosis should include ependynomas, cystic astocytomas, syringomyelis cavities, hydatid cysts, and congenital cysts, Arachnoid and dermoid [11].

\section{CONCLuSion}

Because of human migration, NCC is no longer a rare infection in developed countries. NCC is the most common parasitic disease of the CNS and the most common cause of acquired epilepsy in developing countries. As such, radiologists should be familiar with the radiologic findings of the disease and include it in the differential diagnosis of intracranial lesions, especially in patients from endemic areas. It is therefore vital to have imaging techniques such as MRI in hospitals, both government-run and private-run, in endemic countries for NCC to be eliminated.

\section{ACKNOWLEDGEMENTS}

We are grateful to the Department of Radiology at Ndola Teaching Hospital for sharing with us the MRI images of the patient. We wish to that the Vice-Chancellor of the Copperbelt
University, Kitwe, Zambia, Professor Naison Ngoma, for having supported this work.

\section{REFERENCES}

[1] Carprio A, Escobar A, hauser WA., Cysticercosis and epilepsy: a critical review, Epilepsia.39, 1025-40 (1998).

[2] Garcia HH, Del Brutto OH.,Neurocysticercosis: Updated concepts about an old disease, Lancet Neurol.4, 653-61 (2005).

[3] Wallin MT, Kurtzke JF., Neurocysticercosis in the United States: review of an important emerging infection, Neurology.63, 1559-64 (2004).

[4] Jing-Long Zhao, Alexander Lerner, Zheng Shu, Xing-Jun Gao, Chi-Shing Zee., Imaging spectrum of Nuerocysticercosis, Radiol of Infect Dis.1, 94-102 (2005).

[5] Hawk MW, Shahlaine K, Kim KD, Theis JH., Neurocysticercosis: a review, Surg Neurol. 63, 123-32 (2005).

[6] Estrada S, Verzelli LF, Montilva SS, Acosta CA, Canellas AR. Imaging findings in Neurocysticercosis, Radiol. 55(2), 130-141 (2013).

[7] Dumas JL, Visy JM, Belin C, et al., Parenchymal neurocysticercosis: follow-up and staging MRI, Neuroradiology.39, 12-18 (1997).

[8] Suss RA, Maravilla KR, Thompson J., MR imaging of intracranial cysticercosis: comparison with CT and anatomopathologic features, Am J Neuroradiol. 7, 235-42 (1986).

[9] Del Brutto OH, Rajshekhar V, White AC Jr, et al., Proposed diagnostic criteria for Neurocysticercosis, Neurology. 57, 177-83 (2001).

[10] Garcia HH, Del Bruttto OH., Taenia solium cysticercosis, Infect Dis Clin N Am. 14, 97-119 (2000).

[11] Kimura-Hagama ET, Higuera JA, CoronaCedillo R, Chavez-Macias L, Perochena A, Quiroz-Rojas LY et al., Neurocysticercosis: radiologic-pathologic correlation, Radiographics. 30: 1705-19 (2010).

Citation: Victor Mwanakasale, Nataliya Yakovlyeva, Kapambwe M Kamanga, Pipina V Matafwali. Prompt Magnetic Resonance Imaging, Key to Correct Management of Neurocysticercosis in Developing Countries: A Case Report from Zambia. ARC Journal of Clinical Case Reports. 2020; 6(4):5-8. DOI:https://doi.org/10.20431/2455-9806.0604002.

Copyright: () 2020 Authors. This is an open-access article distributed under the terms of the Creative Commons Attribution License, which permits unrestricted use, distribution, and reproduction in any medium, provided the original author and source are credited. 